\title{
Growth and Magnetic Properties of Fe films on vicinal TO (001) substrates
}

\author{
A.R. Frank, J. Jorzick, M. Rickart, M. Bauer, J. Fassbender, \\ Fachbereich Physik und Forschungsschwerpunkt Materialwissenschaften, Erwin-Schrödinger-Straße 56, Universität Kaiserslautern, 67663 \\ Kaiserslautern, Germany

\section{Scheib} \\ Institut für Oberflächen- und Schichtanalytik GmbH, Universität Kaiserslautern, 67663 Kaiserslautern, Germany
}

S.O. Demokritov*, and B. Hillebrands

\author{
A. Keen, A. Petukhov, A. Kirilyuk, and Th. Rasing \\ Research Institute for Materials University of Nijmegen, The Netherlands
}

\begin{abstract}
We report on investigations of the crystallographic structure and the magnetic anisotropies of epitaxial iron films deposited onto periodically stepped $\mathrm{Ag}(001)$ surfaces using low energy electron diffraction, x-ray diffraction, second harmonic generation (SHG), as well as the Brillouin light scattering (BLS) technique. The focus of the present study lies on the interrelation between the surface morphology of the buffer layers and the magnetic properties of the Fe films, epitaxially grown onto them. Especially the symmetry breaking at the atomic steps is found to create an uniaxial magnetic anisotropy measured by BLS and a very strong anisotropic signal in magnetic SHG.
\end{abstract}

\begin{abstract}
The magnetic properties of ultrathin $3 \mathrm{~d}$ transition metal films have been widely investigated in the last two decades. In recent years it was found that a reduction of the symmetry of these systems by depositing the films onto vicinal cut substrates leads to additional magnetic surface anisotropies [i,ii,iii,iv,v], their magnitude depending on the average step density [vi,vii]. These anisotropies originate from different contributions. Due to the broken symmetry at the step edges the magnetic environment changes. The reduced coordination number and the uniaxial strain induced by the lattice mismatch modificate the band structure and thus the magnetic behavior. A great advantage of these step-induced anisotropies is that they can be artificially controlled by changing the vicinal miscut angle of the substrate, or, in other words, the average step density of the interface or surface [vi,vii]. On the other side these anisotropies can be used in future technical applications because of the artificially controllable character of the step density and so of the magnetic anisotropies.
\end{abstract}

Vicinal to (001) GaAs wafers with fcc Ag-buffers were used in this work as substrates for preparation of bcc Fe-films

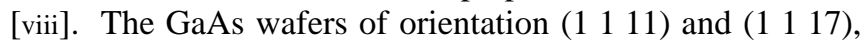
as well as $\left(\begin{array}{lll}0 & 0 & 1\end{array}\right)$-oriented substrates for reference, were chemically cleaned and heated to $870 \mathrm{~K}$ for one hour in ultrahigh vacuum (UHV) to remove the oxides and adsorbates. The base pressure during preparation and deposition was typical lower than $5 \times 10^{-10}$ mbar. To achieve epitaxial growth of the $1500 \AA$ thick fcc Ag-buffer layer the cleaned wafer was covered with a $10 \AA$ thick Fe seed layer. After the deposition the Ag-buffer was annealed at $570 \mathrm{~K}$ for 1 hour to improve the surface morphology. bcc-Fe films of different thicknesses were deposited onto the Ag buffer with a deposition rate of $0.1 \AA / \mathrm{s}$. The first $10 \AA$ of $\mathrm{Fe}$ were grown at room temperature to avoid intermixing at the interface, followed by growth at $\mathrm{T}=370 \mathrm{~K}$. To prevent the samples from oxidization and to insure symmetric interfaces both at the bottom and the top of the Fe-layer, the films were finally covered with $20 \AA$ thick Ag film and $20 \AA$ thick $\mathrm{Cr}$ cap layer.
The chemical cleanliness and the crystallographic structure of the films were controlled in-situ by Auger electron spectroscopy (AES) and low energy electron diffraction (LEED). The AES spectra indicate a typical amount of 5\% or less of a monolayer $\mathrm{Ag}$ on top of the Fe-layer. After annealing the Ag-buffer the LEED images show a crossing of vicinal and lattice reflexes as a function of the electron energy (Fig. 1 a,b). This is a finger print of an ordered step structure on the surface of the Ag-buffer layer with an inclination angle relative to the atomic lattice. The periodicities of the steps [ix] with a terrace width of $11.5 \pm 2 \AA$ and $18.0 \pm 2 \AA$ determined from the LEED data deviate from periodicities resulting from the miscut angle measured by x-ray diffraction (XRD) (see next section) by less than $10 \%$. LEED images taken from these Fe-films show that the energy depending spot splitting vanishes and the spots are elongated along the same direction (Fig. $1 \mathrm{c}, \mathrm{d}$ ).
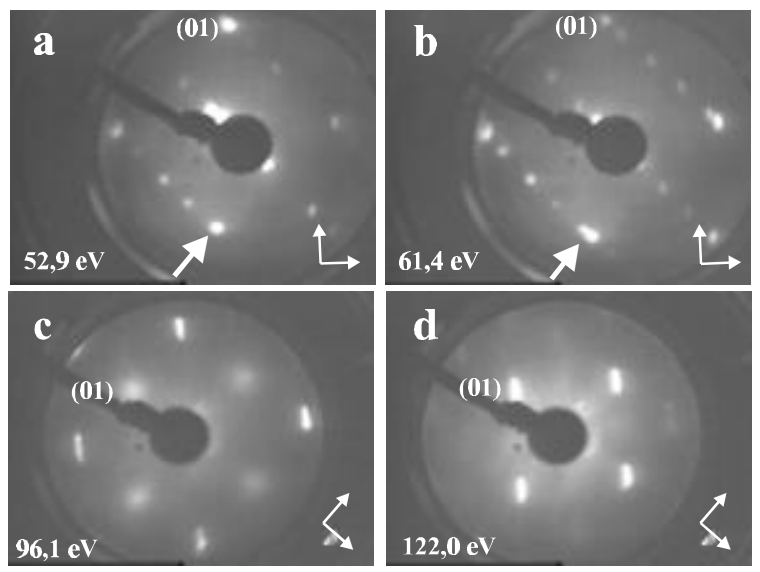

Fig. 1: LEED-images taken from the $\operatorname{Ag}$-buffer $(a, b)$ after annealing at $570 \mathrm{~K}$ showing an energy depending splitting of the spots (electron energy: a) $52.9 \mathrm{eV}$ and b) $61.4 \mathrm{eV}$ ). The step density of the surface determined out of the images is in good agreement with the average step density determined from the XRD data. The images of the Fe-film (c, d) show elongated spots along the step direction. This points to a non-regular step distribution on the surface (electron energy: c) $96.1 \mathrm{eV}$ and b) $122.0 \mathrm{eV}$ ). The (01)-spots of the surface unit cell are marked. The orientation of the sample (indicated by the coordinate system) is indicated. 
This indicates a nonregular step distribution on the surface with an averaged terrace size of approximately $8 \AA$ in the direction perpendicular to the steps.

The vicinal miscut of the substrate, the Ag-buffer layer and a $1000 \AA$ Fe-film was verified by XRD. It was found, that the angles of crystal orientation with respect to the surface normal between the three materials deviate from each other. For the (1 11 17)-oriented sample the vicinal angles of GaAs, $\mathrm{Ag}$ and $\mathrm{Fe}$ are $4.91^{\circ}, 6.09^{\circ}$ and $3.94^{\circ}$ respectively. The angles for the $\left(\begin{array}{lll}1 & 1 & 11\end{array}\right)$ oriented sample are $6.99^{\circ}, 8.75^{\circ}$ and $5.61^{\circ}$. As a matter of fact due to the different crystallographic structure of the substrate and the layers there is an out-of-plane lattice mismatch much larger than the in-plane lattice mismatch. The film-to-film variation of the vicinal angle can be understood by taking this into account $[\mathrm{x}]$ as follows. In Fig. 2 a sketch of a model is shown demonstrating the adoption of the out-of-plane lattice misfit. For simplicity it is assumed that the adoption takes place just within one atomic layer (white layer in Fig. 2). The in-plane lattice mismatch is chosen to be zero. It is seen from Fig. 2 that the [lllll-directions of the lower and the upper layer deviate from each other by an angle $\Delta \alpha$. The angle is given by geometric considerations: $\tan \Delta \alpha=\left(a_{1}\right.$ $\left.a_{2}\right) / t$, where $a_{1,2}$ are the out-of-plane atomic step heights (not to confuse with the lattice constants) and $t$ is the average terrace width. This additional inclination angle can be positive or negative depending on the sign of the out-ofplane misfit. The linear step density $\rho(\rho=1 / t)$ is unchanged if this additional angle is small. This means that the tilt angle between the two materials generates only a small inplane misfit. In the special case of GaAs-Ag and Ag-Fe interfaces the in-plane stress of the misfit parallel to the surface is even reduced. The stress can be reduced in an arbitrary number of layers in both materials depending on the elastic constants. In this way the total energy at the interface is minimized. In comparison with the experimental results we find that the theoretical value of the tilt angle is confirmed with an accuracy better than $5 \%$ for the Ag-Feinterface. For the GaAs-Ag interface the measured value is only $55 \%$ of the theoretical value for both vicinal miscut angles, but the influence of the Fe-interlayer and the nonregular step distribution of the GaAs substrate on the additional miscut is unclear. Also the elastic constants of GaAs are much stiffer than those of the metal films, and the

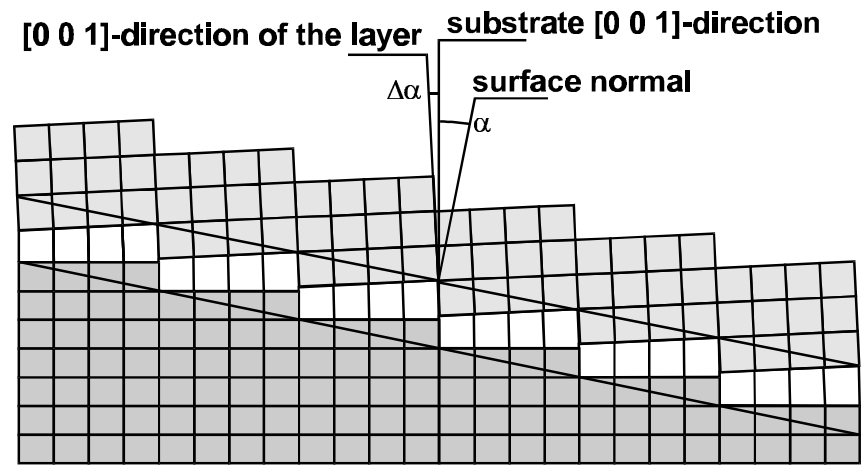

Fig. 2: Two-dimensional model of a vicinal interface generating an additional tilt angle $\Delta \alpha$. This additional angle depends in sign and quantity on the out-of-plane lattice mismatch and the average terrace width between two atomic steps. The [llll 001 -direction of the upper and the lower layer and the direction perpendicular to the interface are displayed. In this figure the deformation is assumed to take place in just one atomic layer. deformation takes place dominantly in the deposited layers. This strain is partly released by dislocation formation in the metal layers.

In order to determine the in-plane and the out-of-plane magnetic anisotropy contributions of twofold and fourfold symmetry, Brillouin light scattering (BLS) from spin waves in the Fe-films was performed. The anisotropy energy is given by:

$$
\begin{aligned}
& \mathrm{E}_{\mathrm{ani}}=-\mathrm{K}_{\mathrm{s}}^{(2)} \cos ^{2} \vartheta+\mathrm{K}_{\mathrm{p}}^{(4)} \sin ^{4} \vartheta \cos ^{2} \varphi \sin ^{2} \varphi \\
& +\mathrm{K}_{\mathrm{p}}^{(2)} \sin ^{2} \vartheta \cos ^{2}\left(\varphi-\varphi_{0}\right)
\end{aligned}
$$

The in- and out-of-plane anisotropy constants $\mathrm{K}_{\mathrm{p}}^{(2)}$, $\mathrm{K}_{\mathrm{s}}^{(2)}$ with twofold symmetry and the in-plane anisotropy constant $\mathrm{K}_{\mathrm{p}}^{(4)}$ with fourfold symmetry were determined by fitting the measured spin wave frequencies as a function of the azimuthal angle $\varphi$ between the [ $\left.\begin{array}{lll}1 & 0 & 0\end{array}\right]$-direction of the $\mathrm{Fe}$ film and the applied external field $H$ with a least squares fit routine.

The details of the fit procedure and the underlying theory are described elsewhere [xi]. Figure 3a shows the measured spin wave frequencies for a set of $20 \AA$ thick Fe-layers as a function of $\varphi$. The maxima in the spin wave frequencies indicate magnetic easy directions. In addition to the fourfold anisotropy, characteristic for the sample without miscut, a
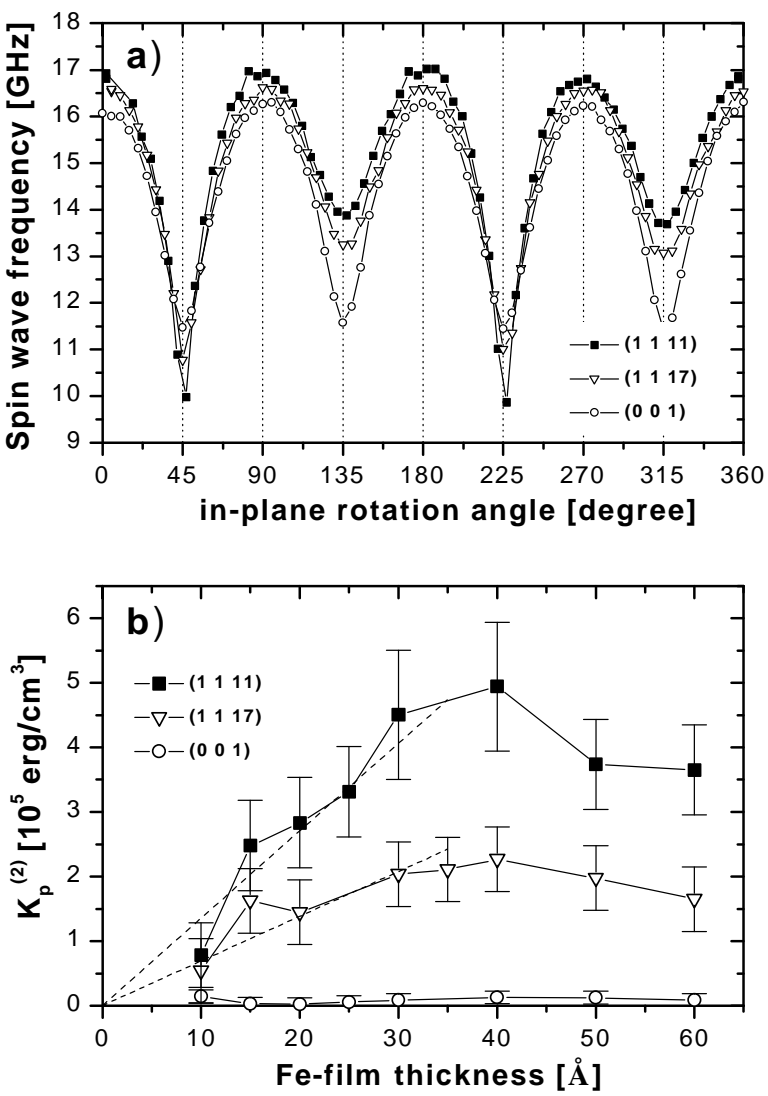

Fig. 3: a) Measured spin wave frequency for three different substrate orientations each with a $20 \AA$ thick Fe-layer as a function of the azimuthal angle $\varphi$. The maxima in the spin wave frequency indicate magnetic easy directions of the layer. The sample without miscut shows a fourfold symmetry. With increasing miscut the additional twofold anisotropy increases. b) The anisotropy constant $\mathrm{K}_{\mathrm{p}}^{(2)}$ as a function of the Fe-film thickness for the three different vicinal angles. The straight lines indicate the initial slopes of the dependencies. The experimental curves show a maximum of the twofold anisotropy at about $40 \AA$. 


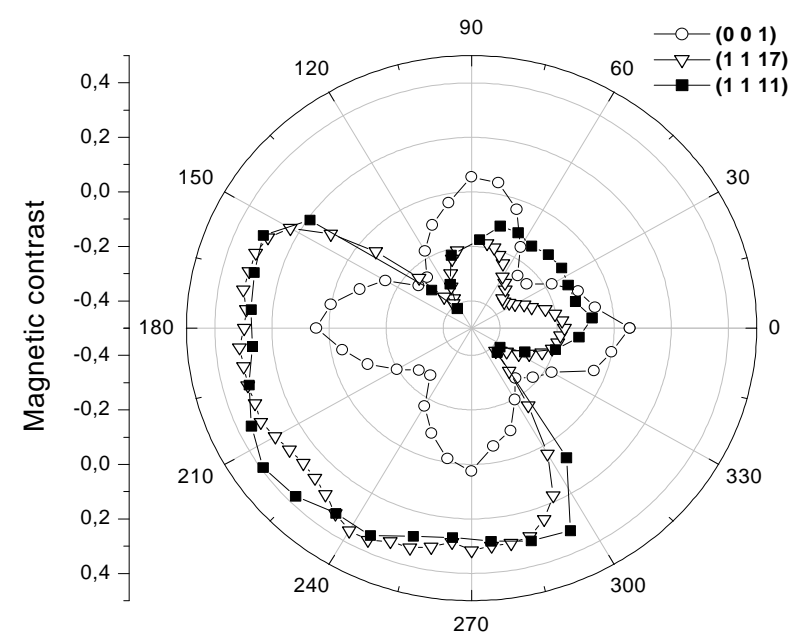

Fig. 4: The magnetic contrast of a set of samples with $30 \AA$ Fe-layer thickness. The polar plot shows a unidirectional symmetry for the two vicinal samples in contrast to the fourfold symmetry of the $\left(\begin{array}{lll}0 & 0 & 1\end{array}\right)$-sample. This represents the high rank of the scattering tensor for vicinal samples.

dominant anisotropy contribution with twofold symmetry is clearly measured for the vicinal samples. This twofold anisotropy is due to the broken symmetry at the surface. Different mechanisms contribute to this anisotropy. First the in-plane anisotropy is determined by the projection of the tensor of the magnetocrystalline anisotropy. This tensor having a cubic symmetry in the crystallographic reference frame is independent of the film thickness and thus leads to a twofold volume anisotropy. Next are the film thickness dependent contributions, which have different origins. Elastic strain (in-plane and out-of-plane) at the interface, especially at the atomic steps, give rise to a magnetoelastic contribution [xii]. In addition the band structure at the interface might be modified due to the reduced coordination number and the chemical environment.

The measurements were performed on the three different orientations of the layers ( $\left.\begin{array}{lll}1 & 1 & 1\end{array}\right), \quad\left(\begin{array}{lll}1 & 1 & 17\end{array}\right)$ and, for reference, $\left(\begin{array}{lll}0 & 0 & 1\end{array}\right)$ within a thickness range from 10 to $60 \AA$ A. While the out-of-plane anisotropy constant $\mathrm{K}_{\mathrm{s}}^{(2)}$ and the inplane anisotropy constant $\mathrm{K}_{\mathrm{p}}^{(4)}$ are almost the same for all orientations, the twofold anisotropy constant $\mathrm{K}_{\mathrm{p}}^{(2)}$ increases for the Fe-film thickness up to $30 \AA$ with increasing film thickness (see Fig. 3b). The slope of the line is roughly proportional to the square of the vicinal angle. At about $40 \AA$ the curves for both miscut angles show a characteristic maximum. This points to a relaxation behavior within the vicinal samples with increasing Fe-film thickness. This can be interpreted as a strain induced anisotropic effect. In

References:

[i] M. Albrecht, T. Furubayashi, M. Przybylski, J. Korecki, U. Gradmann, J. Magn. Magn. Mater. 113, 207 (1992).

[ii] A. Berger, U. Linke, H.P. Oepen, Phys. Rev. Lett. 68, 839 (1992).

[iii] J. Chen, J.L. Erskine, Phys. Rev. Lett. 68, 1212 (1992).

[iv] P. Krams, F. Lauks, R.L. Stamps, B. Hillebrands, G. Güntherodt, H.P. Oepen, J. Magn. Magn. Mater. 121, 483 (1992).

[v] U. Gradmann, J. Korecki, G. Waller, Appl. Phys. A, 39,101 (1986).

[vi] R. K. Kawakami, E.J. Escorcia-Aparicio, Z.Q. Qiu, Phys. Rev. Lett. 77, 2570 (1996).

[vii]R. K. Kawakami, M.O. Bowen, H.J. Choi, E.J. Escorcia-Aparicio, Z.Q. Qiu, Phys. Rev. B. 58, R5924 (1998). combination with the additional miscut angle between the $\mathrm{Ag}$ and the Fe-layer we estimate that the out-of-plane lattice mismatch generates this anisotropy contribution, but with increasing thickness of the Fe-layer this stress is reduced or vanishes completely. This leads to a decreasing behavior of the twofold anisotropy constant. Generally the twofold anisotropy is of one order of magnitude larger than the values one would expect from the rotation of the magnetocrystalline tensor. This means that the anisotropy is likely to be a step induced anisotropy of the Fe-film. This result is confirmed by BLS measurements performed on $1000 \AA$ thick Fe-layers displaying no twofold anisotropy contribution for all crystal orientations within the measurement accuracy.

Symmetry breaking at atomic steps on vicinal surfaces and interfaces make this system an ideal candidate for investigation with the magnetization induced second harmonic generation (MSHG) technique [xiii]. This technique is a nonlinear magneto-optical tool sensitive to both structural and magnetic properties of surfaces and interfaces. The strong surface/interface sensitivity is derived from the break in symmetry at boundaries between centrosymmetric media. The technique is non-invasive and therefore particularly suited for the study of buried interfaces. Measurements have been performed using a mode-locked Ti:sapphire laser operating at a repetition rate of $76 \mathrm{MHz}$ with a pulse width of about $100 \mathrm{fs}$ and a wavelength of $800 \mathrm{~nm}$. The laser fluence was regulated such that the average laser power on the sample was $50 \mathrm{~mW}$. The MSHG data were recorded in the transversal magnetic configuration at an angle of light incidence of $45^{\circ}$. After filtering the outgoing specular second-harmonic light, in order to suppress the fundamental frequency, the light was detected with a cooled photomultiplier as a function of the azimuthal rotation of the sample. Figure 4 demonstrates typical MSHG patterns from the $30 \AA \mathrm{Fe}$-film for PPpolarization combinations. Shown is the magnetic contrast $\rho$, defined by the difference of the MSHG-signal for two opposite field directions divided by the sum of the two signals. These rotational anisotropy patterns shows a very distinct asymmetry in the lobe structure and a 1-fold symmetry due to the vicinal nature of the Fe/Ag interface.

Support by the Deutsche Forschungsgemeinschaft, the European Science Foundation (Project NANOMAG) and the European TMR network NOMOKE (contract no. ERBFMRXCT 960015) is gratefully acknowledged.

[viii] B.F.P. Roos, A.R. Frank, S.O. Demokritov, B. Hillebrands, J. Magn. Magn. Mater. 198-199, 725 (1992).

[ix] M. Henzler, Appl. Phys. 9, 11 (1976).

[x] H. Nagai, J. Appl. Phys. 45, 3789 (1974).

[xi] B. Hillebrands, Phys. Rev. B 41,530 (1990).

[xii]D. Sander Rep. Prog. Phys. 62, 809 (1999).

[xiii] Th. Rasing, Appl. Phys. B 68, 477 (1999). 